\title{
Residual Effect of Levels of Biochar and FYM on Growth, Yield and Nutrient Uptake by Green Gram (Vigna radiate L.) Crop in Sandy Loam Soil
}

\author{
B. R. Arunkumar* and G. N. Thippeshappa \\ Department of Soil Science and Agricultural Chemistry, College of Agriculture, University of \\ Agricultural and Horticultural Sciences, Navile, Shivamogga, Karnataka, India-577225 \\ *Corresponding author
}

\begin{tabular}{|l|}
\hline Ke y w o r d s \\
Biochar, Residual, \\
Grain yield, \\
Nutrient uptake \\
\hline Article Info \\
\hline Accepted: \\
08January 2020 \\
Available Online: \\
10 February 2020
\end{tabular}

\section{Keywords}

Biochar, Residual, Grain yield, Nutrient uptake

Article Info

Accepted:

Available Online:

10 February 2020

\section{A B S T R A C T}

A Field experiment was conducted at Zonal Agricultural and Horticultural Research Station, Shivamogga, Karnataka to see the residual effect of biochar and FYM on productivity of succeeding green gram crop during Kharif 2018 after harvest of summer aerobic rice crop. Results revealed that the growth and yield attributing characters of green gram viz., plant height and number of leaves per plant, number of pods bearing auxiliary branches per plant, pod length, number of pods per plant, and number of seeds per pod were significantly influenced by residual effect applied biochar and FYM with RDF at different growth stages. However, higher growth parameters were observed in treatment contains CS-biochar $8 \mathrm{t} \mathrm{ha}^{-1}$ and $10 \mathrm{t} \mathrm{ha}^{-1} \mathrm{FYM}$ with RDF $\left(\mathrm{T}_{16}\right)$ compared to RDF alone and absolute control. Green gram seed and stover yields were significantly influenced by the residual levels of biochar and FYM combinations. Higher seed and stover yield (871.00 and $1708 \mathrm{~kg} \mathrm{ha}^{-1}$ ) was recorded in CS-biochar @ 8t ha ${ }^{-1}+\mathrm{FYM} 10 \mathrm{t} \mathrm{ha}^{-1}+\mathrm{RDF}\left(\mathrm{T}_{16}\right)$ treatment and which showed the percentage efficiency of 4.72 to 14.30 per cent and 4.90 to 14.63 per cent increase in seed and stover yield of a green gram as compared to the treatment, $\mathrm{T}_{4}\left(\mathrm{RDF}+\mathrm{FYM} 10 \mathrm{t} \mathrm{ha}^{-1}\right)$. Similarly primary, secondary and micronutrients uptake by green gram plant at harvest varied significantly due to the residual levels of biochar and FYM with RDF. However, the highest content and uptake of these nutrients was observed in treatment, $\mathrm{T}_{16}$ (CS-biochar@8t ha $\left.{ }^{-1}+\mathrm{FYM} 10 \mathrm{t} \mathrm{ha}^{-1}+\mathrm{RDF}\right)$ as compared to other treatments.

\section{Introduction}

In recent years, biochar has emerged as an organic amendment with mineral nutrient elements and hold a promise to improve the soil quality and yield of crops. The biochar is found to have a positive impact on soil fertility, resulting in an increase in crop yield without causing a hazard to soil and water environment. Biochar produced from varied technological methods of pyrolysis can convert agriculture crop residues like coconut shells, arecanut husks, maize cob, cerealpulse crop husks, grasses, forestry products, 
animal and poultry manures to biochar. The biochar consists mainly $\mathrm{C}$, and contains $\mathrm{O}, \mathrm{H}$, $\mathrm{N}$ and ash (calcium $(\mathrm{Ca})$, potassium $(\mathrm{K})$, etc.,).

Biochar is a fine grained, highly porous charcoal substance that is distinguished from other charcoals in its intended use as a soil amendment. The particular heat treatment of organic biomass used to produce biochar contributes to its large surface area and its characteristic ability to persist in soils with very little biological decomposition (Lehmann et al., 2006). Biochar serves as a catalyst that enhances plant uptake of nutrients and water. Compared to other soil amendments, the high surface area and porosity of biochar makes it to adsorb or retain nutrients and hold moisture and in addition to this labile fraction of $\mathrm{C}$ in biochar provides $\mathrm{C}$ and energy to heterotrophic beneficial microorganisms to flourish and the ash fraction may supply some of the mineral nutrient requirements for crops (Glaser et al., 2002, and Warnock et al., 2007). As a soil amendment, biochar has been proven to have many positive effects in improving soil quality (2). Biochar application had been proven able to improve soil physico-chemical properties, fertility and biological activity and increased crop yield. The residual biochar application to soil as organic nutrient source and its efficacy in increase crop yield has been shown by Widowati et al., (2017). The increase of crop yield with biochar application has been reported elsewhere, such as for rice, maize, cassava, soybean, and cowpea (Wisnubroto et al., 2016).

Green gram is one of the important legume or pulse crop Indian diet. It grown largely in cereal -pulse based cropping system where cereals as first crop fallowed by green gram as succeeding pulse crop. The yields green gram stagnated in recent years with the nutrient and moisture stress as this crop normally grown in stored soil moisture and preceding soil nutrients. Hence, the strategy for higher yields in the green gram should be formulated using the combined application of organics, inorganics and biofertilizers coupled with the inclusion of crops in summer fallows for sustainable yields and preservation of soil health.

Biochar application to soil may thus improve the physical properties of soil because of retardation of native stable organic matter decomposition. It persists for a longer time in soil. Therefore, it is indeed to study the residual effect of biochar on growth and yield of succeeding crop. The studies on effects of biochar application on soil properties especially in aerobic soils or its potentiality as a nutrient source are very scanty and it deserves detailed investigation. The information from this reported experiment will be very helpful to pulse growing farmers, because it will help them in soil fertility management for their crops.

\section{Materials and Methods}

An experiment was established in the Zonal Agricultural and Horticultural Research Station, Navile, Shivamogga, coming under University of Agricultural and Horticultural Sciences, Shivamogga which belongs to Southern Transition Agro-climatic Zone (Zone - 7) of Karnataka. The research station is situated at $14^{0} 0^{\prime}$ ' to $14^{0} 1^{\prime}$ 'North latitude and $75^{\circ} 40^{\prime}$ to $75^{\circ} 42^{\prime}$ east longitude with an altitude of 650 meters above the mean sea level. The average rainfall of the zone is $873.40 \mathrm{~mm}$. Initial soil characterization of experimental site indicated that soil had a bulk density of $1.73 \mathrm{Mg} \mathrm{m}^{-3}$, maximum water holding capacity of 24.58 per cent, field capacity of 11.80 per cent and $\mathrm{pH}$ of $5.88, \mathrm{EC}$ of $0.22 \mathrm{dS} \mathrm{m}^{-1}$ with the organic carbon content of $4.68 \mathrm{~g} \mathrm{~kg}^{-1}$ and CEC of $14.43 \mathrm{cmol}(\mathrm{p}+)$ $\mathrm{kg}^{-1}$. Further, the soil was low in available 
nitrogen $\left(213.00 \mathrm{~kg} \mathrm{ha}^{-1}\right)$, high in available phosphorus status $\left(58.00 \mathrm{~kg} \mathrm{ha}^{-1}\right)$ and medium in available potassium status $\left(158.00 \mathrm{~kg} \mathrm{ha}^{-1}\right)$. The exchangeable $\mathrm{Ca}$ and $\mathrm{Mg}$ were 2.85 and $1.74\left(\mathrm{cmol}\left(\mathrm{p}^{+}\right) \mathrm{kg}^{-1}\right)$, available sulphur was $11.59 \mathrm{mg} \mathrm{kg}^{-1}$ and all the DTPA extractable micronutrients were above the critical limits (Fe - 12.18, $\mathrm{Mn}-2.58, \mathrm{Zn}-2.18$ and $\mathrm{Cu}-1.13$ $\mathrm{mg} \mathrm{kg}{ }^{-1}$ ). The soil belongs to the taxonomic class Typic haplustalf with sandy loam texture. Experimental site before used for this study, the soil was used for biochar experiment on aerobic rice crop during summer 2018 with 16 treatments consisting of four levels of coconut shell biochar at 2, 4, 6 and $8 \mathrm{tha}^{-1}$ and two levels of FYM at 5 and 10 $\mathrm{t} \mathrm{ha}^{-1}$ which were applied alone and in combinations to soil with recommended dose of fertilizer (100:50:50 kg ha-1) was applied commonly to all the treatments except absolute control. The treatments were imposed in randomized complete block design (RCBD) design with three replications for each treatment. The second experiment was conducted immediately after the harvest of aerobic rice in the same plots of the first experiment, using pulse crop (green gram). The test crop was grown to know the residual effect of different levels of biochar and FYM on growth and yield of succeeding green gram crop in the same treatment combination and layout of the earlier experiment. The only recommended dose of fertilizer NPK at 20:50:50 kg ha ${ }^{-1}$ was added to all treatments except treatment $\mathrm{T}_{1}$ (absolute control).

After harvest of first crop (Aerobic rice) land was fallow for nearly one month. Then each plot is manually digged without damage to bunds and weeds were removed, made the soil into fine tilth for sowing crop. The recommended dose of nitrogen, phosphorus and potassium were applied as urea, DAP and muriate of potash during the sowing time. The green gram seeds were sown in each plot with a spacing of $30 \mathrm{~cm}$ in between the rows and
$10 \mathrm{~cm}$ in between the plants during Kharif 2018. Observations on growth and yield parameters were recorded on randomly selected five plants from each net plot and tagged for recording the observations. The experimental data obtained were subjected to statistical analysis adopting Fisher's method of analysis of variance as out lined by Gomez and Gomez (1984). The level of significance used in the $\mathrm{F}$ test was at 5 per cent. Critical difference $(\mathrm{CD})$ values are given for the data at 5 per cent level of significance, wherever the $\mathrm{F}$ test was significant.

\section{Results and Discussion}

\section{Growth parameters}

Plant height differed significantly by applied different levels of biochar and FYM at all the growth stages, except at 30 DAS (Table 1). At 45 DAS, significantly higher plant height was recorded in the treatment $T_{16}$ which, received that CS-biochar@8 t ha ${ }^{-1}+$ FYM @10 t $\mathrm{ha}^{-1}+\operatorname{RDF}(29.19 \mathrm{~cm})$ followed by the treatment, $\mathrm{T}_{15}$ (CS-biochar @ $6 \mathrm{t} \mathrm{ha}^{-1}+\mathrm{FYM}$ @ $10 \mathrm{t} \mathrm{ha}^{-1}+\mathrm{RDF}$ ) which recorded $27.78 \mathrm{~cm}$ and it was on par with CS-biochar @ $4 \mathrm{t} \mathrm{ha}^{-1}$ + FYM @ $10 \mathrm{t} \mathrm{ha}^{-1}+\mathrm{RDF}\left(\mathrm{T}_{14}\right)(26.15 \mathrm{~cm})$ as compared to $\mathrm{T}_{4}\left(\mathrm{RDF}+\mathrm{FYM} @ 10 \mathrm{t} \mathrm{ha}^{-1}\right)$ and RDF alone treatment (22.90 and 20.43 $\mathrm{cm}$, respectively) and remaining treatments were on par with each other. Significantly taller plant at harvest was noticed in the treatments with residual effect of CS-biochar @ $8 \mathrm{t} \mathrm{ha}^{-1}+\mathrm{FYM} @ 10 \mathrm{t} \mathrm{ha}^{-1}+\mathrm{RDF}\left(\mathrm{T}_{16}\right)$ $(31.10 \mathrm{~cm})$ it was followed by CS-biochar @ $6 \mathrm{tha}^{-1}+$ FYM @ $10 \mathrm{tha}^{-1}+\operatorname{RDF}(29.14 \mathrm{~cm})$ which was on par with CS-biochar@ $4 \mathrm{t} \mathrm{ha}^{-1}$ +FYM@10 t ha ${ }^{-1}+$ RDF $(28.48 \mathrm{~cm})$ as compared to $\mathrm{T}_{4}\left(\mathrm{RDF}+\mathrm{FYM} @ 10 \mathrm{t} \mathrm{ha}^{-1}\right)$ and RDF alone treatment (23.41 and 22.64 $\mathrm{cm}$, respectively). Numbers of leaves differed significantly by levels of residual effect of biochar and FYM at all the growth stages except at 30 DAS (Table 1). 
Number of leaves per plant at 45 DAS, significantly influenced by residual effect of applied levels of biochar and FYM and their combination over absolute control and RDF treatments. Higher number of leaves per plant (25.94) was observed due to application of CS-biochar@8 t ha ${ }^{-1}$ +FYM@10 t ha ${ }^{1}\left(\mathrm{~T}_{16}\right)$ followed by CS-biochar@6 t ha ${ }^{-1}+$ FYM @ $10 \mathrm{t} \mathrm{ha}^{-1}(23.52)$ over all other treatments. Even at harvest stage of crop, the treatment, $\mathrm{T}_{16}$ (CS-biochar@8 t ha ${ }^{-1}+$ FYM @ 10

$\mathrm{t}$

$\mathrm{ha}^{-1}$ ) registered significantly higher number of leaves per plant (26.91) which was on par with CS-biochar@6 t ha ${ }^{-1}$ +FYM @ 10 t $\mathrm{ha}^{-1}$ (24.62) as compared to $\mathrm{T}_{4}$ (POP) and RDF alone treatment (19.44 and 16.68, respectively).

Plant height and number of leaves were significantly increased by the imposed treatments at all stages of crop growth. It might be attributed to the residual effect of biochar providing better growing conditions to crop by continuous supply of nutrients and improvement of soil properties. The variation in plant height was considered to be due to variation in the availability of major nutrients. Increase in the effective microorganisms in the soils increases the mineralization. This was supported by Muthaura et al., (2010). Mirl et al., (2013) observed an increase in plant height and number of leaves with FYM treatment as compared to without biochar. Similar increase in crop growth with combined application of biochar and FYMwas observed by Bandara et al., (2015).

\section{Yield parameters and yield}

Data on yield parameters of green gram at harvest viz., number of pod bearing auxiliary branches per plant, pod length, number of pods per plant, number of seeds per pod, test weight (first and second picking), seed and stover yield as influenced by the residual effect of biochar and FYM are presented in Table 2. Residual effect of CS-biochar@8 t $\mathrm{ha}^{-1}$ + FYM @10 t ha ${ }^{-1}$ + RDF has resulted significantly higher number of pod bearing auxiliary branches per plant (4.38) followed by CS-biochar@6 tha ${ }^{-1}$ FYM@10 t ha ${ }^{-1}$ + RDF (4.26) which was on par with CSbiochar@4 tha $\mathrm{ha}^{-1}+\mathrm{FYM} @ 10 \mathrm{t} \mathrm{ha} \mathrm{h}^{-1}+\mathrm{RDF}$ (4.22) as compared to $\mathrm{T}_{4}$ (POP) and RDF alone treatment (3.0 and 2.45, respectively). Pod length and number of seeds per pod was found to be non-significant due to residual effect of biochar and FYM. However, pod length and number of seeds per pod varied from 5.44 to $8.50 \mathrm{~cm}$ and 8.30 to 14.36 seeds per pod, respectively.

Significant differences in number of pods per plant were found due to residual effect of applied levels of biochar and FYM (Table 2). Significantly a greater number of pods per plant (25.57) obtained in the treatments of CS-biochar@8 t ha ${ }^{-1}$ +FYM @10 t ha ${ }^{-1}$ followed by CS-biochar@6 tha ${ }^{-1}$ +FYM @ $10 \mathrm{t} \mathrm{ha}^{-1}$ (24.71) over other treatments. However, significantly lower number of pods per plant (16.41) was recorded in the treatment, $\mathrm{T}_{1}$ (absolute control). The test weight of green gram seeds was not influenced significantly due to treatments during first and second picking. During first picking test weight ranges from $4.13 \mathrm{~g}$ in residual effect of CS-biochar @ $2 \mathrm{t} \mathrm{ha}^{-1}$ to $4.50 \mathrm{~g}$ in CS-biochar@8 t ha ${ }^{-1}+$ FYM @ 10 $\mathrm{t} \mathrm{ha}^{-1}+\mathrm{RDF}$ treatments. Whereas, in second picking it ranges from $3.95 \mathrm{~g}$ in absolute control to 4.32 g CS-biochar@ @ t ha ${ }^{-1}+$ FYM@10 $\mathrm{tha}^{-1}+$ RDF treatments.

The data showed similar trend with respect to seed and stover yields. Seed and stover yields of green gram was significantly influenced by the residual effect of levels of biochar and FYM and their combined application (Table 2). 
Table.1 Residual effect of levels of biochar and FYM on plant growth parameters at different growth stages of green gram

\begin{tabular}{|c|c|c|c|c|c|c|}
\hline \multirow[t]{2}{*}{ Treatments } & \multicolumn{3}{|c|}{ Plant height (cm) } & \multicolumn{3}{|c|}{ Number of leaves plant ${ }^{-1}$} \\
\hline & 30 DAS & 45 DAS & Harvest & 30 DAS & 45 DAS & Harvest \\
\hline T1 & 11.22 & 15.18 & 19.63 & 10.52 & 13.57 & 12.62 \\
\hline $\mathbf{T 2}$ & 14.13 & 20.43 & 22.64 & 13.48 & 17.67 & 16.68 \\
\hline T3 & 14.23 & 21.17 & 22.85 & 14.74 & 18.49 & 19.06 \\
\hline T4 & 14.53 & 22.90 & 23.41 & 15.40 & 18.53 & 19.44 \\
\hline T5 & 14.49 & 20.75 & 22.41 & 13.59 & 17.89 & 17.80 \\
\hline T6 & 14.28 & 21.37 & 22.26 & 14.85 & 18.54 & 18.56 \\
\hline T7 & 14.63 & 22.16 & 23.49 & 14.47 & 19.10 & 19.67 \\
\hline T8 & 14.68 & 22.90 & 23.82 & 14.89 & 19.55 & 19.61 \\
\hline T9 & 14.30 & 23.92 & 24.67 & 13.66 & 18.32 & 18.48 \\
\hline T10 & 14.75 & 24.47 & 25.69 & 14.11 & 18.49 & 18.60 \\
\hline T11 & 14.88 & 24.62 & 25.71 & 15.01 & 19.66 & 20.56 \\
\hline T12 & 15.53 & 25.67 & 27.18 & 16.21 & 20.32 & 21.61 \\
\hline T13 & 15.25 & 25.73 & 27.09 & 15.16 & 21.55 & 22.46 \\
\hline T14 & 15.44 & 26.15 & 28.48 & 15.27 & 21.87 & 22.86 \\
\hline T15 & 15.68 & 27.78 & 29.14 & 16.25 & 23.52 & 24.62 \\
\hline T16 & 16.93 & 29.19 & 31.10 & 16.86 & 25.94 & 26.91 \\
\hline S.Em \pm & 1.10 & 1.16 & 0.95 & 1.09 & 1.13 & 1.26 \\
\hline C.D. $(p=0.05)$ & NS & 3.35 & 2.74 & NS & 3.27 & 3.63 \\
\hline
\end{tabular}

Note: CS: Coconut shell, RDF: Recommended dose of fertilizer, POP: Package of practice

\begin{tabular}{|c|c|c|}
\hline$T_{1}$ : Absolute Control & $\mathrm{T}_{7}$ : CS - Biochar @ $6 \mathrm{t} \mathrm{ha}^{-1}$ & $\mathrm{~T}_{12}$ : CS - Biochar @8 tha $\mathrm{ha}^{-1}+\mathrm{FYM} @ 5 \mathrm{tha}^{-1}$ \\
\hline$T_{2}: 100: 50: 50 \mathrm{NPK} \mathrm{kg} \mathrm{ha}^{-1}$ (Only RDF) & $\mathrm{T}_{8}: \mathrm{CS}$ - Biochar @ $8 \mathrm{t} \mathrm{ha}^{-1}$ & $\mathrm{~T}_{13}:$ CS - Biochar@ $2 \mathrm{tha}^{-1}+\mathrm{FYM} @ 10 \mathrm{tha}^{-1}$ \\
\hline$T_{3}: F Y M @ 5$ tha $^{-1}$ & $\mathrm{~T}_{9}$ : CS - Biochar@ $2 \mathrm{tha}^{-1}+\mathrm{FYM} @ 5 \mathrm{tha}^{-1}$ & T14: CS - Biochar@ $4 \mathrm{tha}^{-1}+\mathrm{FYM} @ 10 \mathrm{tha}^{-1}$ \\
\hline $\mathrm{T}_{4}$ : FYM@10 $\mathrm{tha}^{-1}(\mathrm{POP})$ & $\mathrm{T}_{10}$ : CS - Biochar@ $9 \mathrm{t} \mathrm{ha}^{-1}+\mathrm{FYM} @ 5 \mathrm{tha}^{-1}$ & T15: CS - Biochar@6 tha ${ }^{-1}+$ FYM @ $10 \mathrm{tha}^{-1}$ \\
\hline $\mathrm{T}_{5}: \mathrm{CS}$ - Biochar @ $2 \mathrm{t} \mathrm{ha}^{-1}$ & $\mathrm{~T}_{11}$ : CS - Biochar @ $6 \mathrm{t} \mathrm{ha}^{-1}+\mathrm{FYM} @ 5 \mathrm{tha}^{-1}$ & $\mathrm{~T}_{16}:$ CS - Biochar@8 $\mathrm{tha}^{-1}+\mathrm{FYM} @ 10 \mathrm{tha}^{-1}$ \\
\hline $\mathrm{T}_{6}:$ CS - Biochar @ $4 \mathrm{t} \mathrm{ha}^{-1}$ & & \\
\hline
\end{tabular}


Table.2 Residual effect of levels of biochar and FYM on yield parameters and yield of green gram at harvest

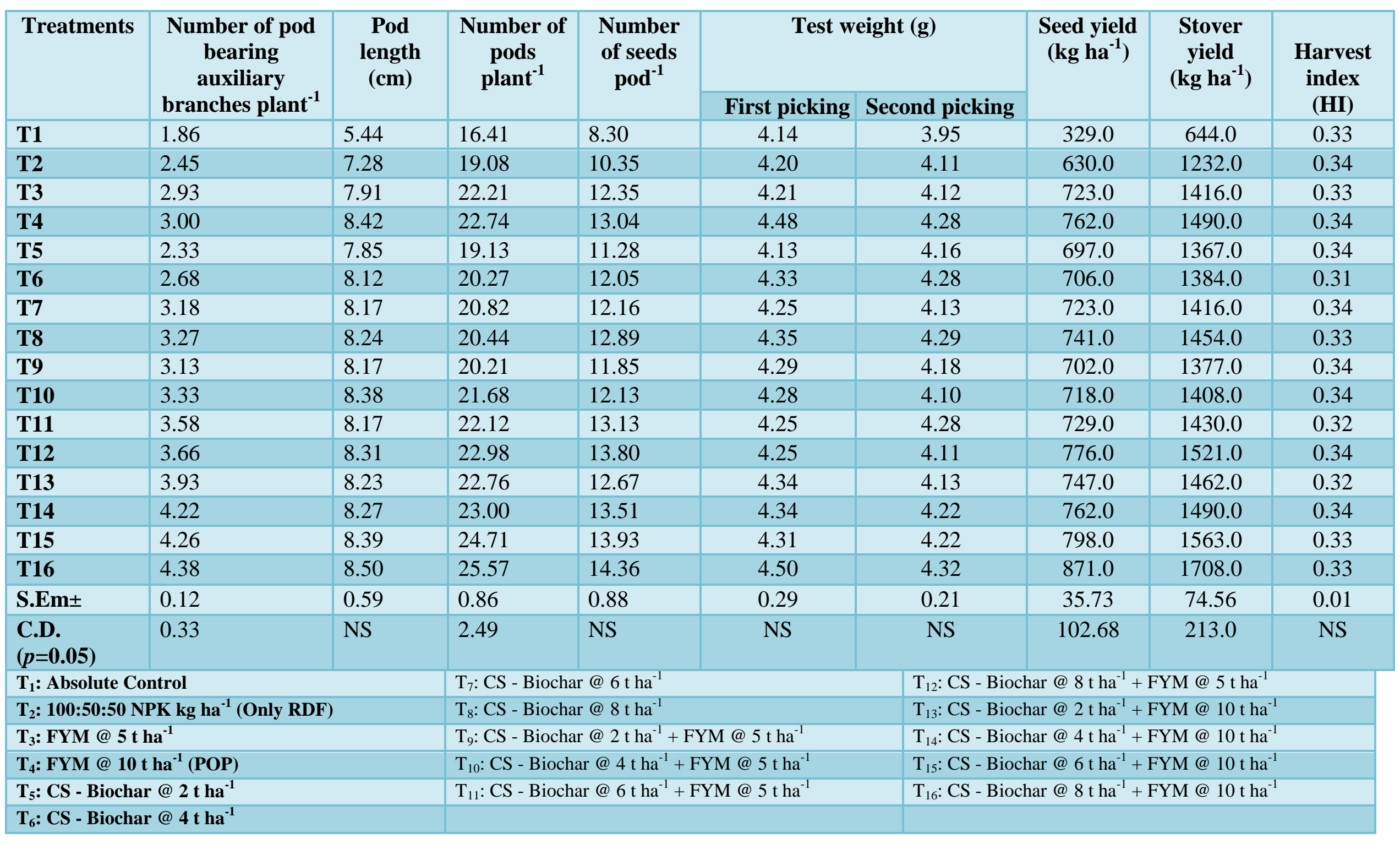


Table.3 Residual effect of levels of biochar and FYM on uptake of primary nutrients by seed and stover of green gram at harvest

\begin{tabular}{|c|c|c|c|c|c|c|c|c|c|}
\hline \multirow[t]{2}{*}{ Treatments } & \multicolumn{3}{|c|}{$\begin{array}{c}\mathrm{N} \\
\left(\mathrm{kg} \mathrm{ha}^{-1}\right)\end{array}$} & \multicolumn{3}{|c|}{$\begin{array}{c}\mathbf{P} \\
\left(\mathrm{kg} \mathrm{ha}^{-1}\right)\end{array}$} & \multicolumn{3}{|c|}{$\begin{array}{c}\mathrm{K} \\
\left(\mathrm{kg} \mathrm{ha}^{-1}\right)\end{array}$} \\
\hline & Seed & Stover & Total & Seed & Stover & Total & Seed & Stover & Total \\
\hline T1 & 11.71 & 7.21 & 18.93 & 0.56 & 0.64 & 1.20 & 2.04 & 5.67 & 7.71 \\
\hline $\mathbf{T} 2$ & 26.52 & 51.99 & 78.51 & 1.64 & 2.83 & 4.47 & 7.75 & 13.80 & 21.55 \\
\hline T3 & 37.31 & 31.86 & 69.17 & 2.17 & 3.82 & 5.99 & 9.76 & 16.71 & 26.47 \\
\hline T4 & 41.00 & 33.97 & 74.97 & 2.36 & 4.32 & 6.68 & 10.52 & 18.03 & 28.54 \\
\hline T5 & 30.46 & 29.12 & 59.58 & 1.74 & 3.01 & 4.75 & 8.71 & 15.58 & 24.30 \\
\hline T6 & 33.11 & 31.42 & 64.53 & 1.77 & 2.77 & 4.53 & 8.83 & 16.33 & 25.16 \\
\hline $\mathbf{T 7}$ & 37.02 & 31.58 & 68.59 & 1.59 & 2.41 & 4.00 & 9.18 & 16.99 & 26.17 \\
\hline T8 & 38.75 & 33.44 & 72.20 & 1.70 & 2.62 & 4.32 & 9.56 & 17.59 & 27.15 \\
\hline T9 & 32.36 & 30.29 & 62.66 & 1.61 & 2.48 & 4.09 & 9.13 & 15.28 & 24.41 \\
\hline T10 & 35.54 & 31.12 & 66.66 & 1.72 & 2.82 & 4.54 & 9.41 & 16.90 & 26.30 \\
\hline T11 & 37.62 & 32.03 & 69.65 & 2.19 & 4.00 & 6.19 & 9.70 & 17.16 & 26.86 \\
\hline T12 & 41.90 & 34.83 & 76.73 & 2.64 & 4.41 & 7.05 & 10.32 & 18.56 & 28.88 \\
\hline T13 & 39.44 & 33.92 & 73.36 & 2.32 & 4.09 & 6.41 & 10.38 & 17.84 & 28.22 \\
\hline T14 & 41.68 & 36.13 & 77.81 & 2.51 & 4.63 & 7.14 & 10.90 & 18.51 & 29.41 \\
\hline T15 & 46.12 & 40.33 & 86.45 & 2.79 & 5.16 & 7.95 & 11.97 & 20.32 & 32.29 \\
\hline T16 & 51.82 & 44.58 & 96.40 & 3.31 & 5.81 & 9.12 & 13.76 & 23.40 & 37.16 \\
\hline S.Em \pm & 1.24 & 1.55 & 2.10 & 0.09 & 0.19 & 0.22 & 0.43 & 0.68 & 0.81 \\
\hline C.D. $(p=0.05)$ & 3.59 & 4.47 & 6.06 & 0.26 & 0.55 & 0.65 & 1.25 & 1.97 & 2.35 \\
\hline
\end{tabular}

Note: CS: Coconut shell, RDF: Recommended dose of fertilizer, POP: Package of practice

\begin{tabular}{|c|c|c|}
\hline $\mathbf{T}_{1}$ : Absolute Control & $\mathrm{T}_{7}: \mathrm{CS}$ - Biochar @ $6 \mathrm{tha}^{-1}$ & $\mathrm{~T}_{12}: \mathrm{CS}$ - Biochar@8 $\mathrm{t} \mathrm{ha} \mathrm{a}^{-1}+\mathrm{FYM} @ 5 \mathrm{t} \mathrm{ha}^{-1}$ \\
\hline $\begin{array}{l}T_{2}: 100: 50: 50 \mathrm{NPK} \mathrm{kg} \mathrm{ha}^{-1} \text { (Only } \\
\text { RDF) }\end{array}$ & T8: CS - Biochar@ $8 \mathrm{t} \mathrm{ha}^{-1}$ & T 13 : CS - Biochar@ $2 \mathrm{t} \mathrm{ha}^{-1}+\mathrm{FYM} @ 10 \mathrm{t} \mathrm{ha}^{-1}$ \\
\hline T $_{3}:$ FYM @ 5 tha $^{-1}$ & $\mathrm{~T}_{9}: \mathrm{CS}$ - Biochar @ $2 \mathrm{tha}^{-1}+\mathrm{FYM} @ 5 \mathrm{tha}^{-1}$ & T14: CS - Biochar @ $4 \mathrm{t} \mathrm{ha}^{-1}+\mathrm{FYM} @ 10 \mathrm{t} \mathrm{ha}^{-1}$ \\
\hline $\mathrm{T}_{4}:$ FYM @ $10 \mathrm{t} \mathrm{ha}^{-1}$ (POP) & $\mathrm{T}_{10}$ : CS - Biochar@4 tha ${ }^{-1}+\mathrm{FYM} @ 5 \mathrm{tha}^{-1}$ & $\mathrm{~T}_{15}: \mathrm{CS}$ - Biochar@6 tha $\mathrm{t}^{-1}+\mathrm{FYM} @ 10 \mathrm{t} \mathrm{ha}^{-1}$ \\
\hline $\mathrm{T}_{5}: \mathrm{CS}$ - Biochar@ $2 \mathrm{t} \mathrm{ha}{ }^{-1}$ & $\mathrm{~T}_{11}$ : CS - Biochar@6 tha ${ }^{-1}+\mathrm{FYM} @ 5 \mathrm{tha}^{-1}$ & T16: CS - Biochar@8 $\mathrm{tha}^{-1}+\mathrm{FYM} @ 10 \mathrm{t} \mathrm{ha}^{-1}$ \\
\hline $\mathrm{T}_{6}: \mathrm{CS}$ - Biochar@ $4 \mathrm{t} \mathrm{ha}^{-1}$ & & \\
\hline
\end{tabular}


Table.4 Residual effect of levels of biochar and FYM on uptake of secondary nutrients by seed and stover of green gram at harvest

\begin{tabular}{|c|c|c|c|c|c|c|c|c|c|}
\hline \multirow[t]{2}{*}{ Treatments } & \multicolumn{3}{|c|}{$\begin{array}{c}\text { Ca } \\
\left(\mathrm{kg} \mathrm{ha}^{-1}\right)\end{array}$} & \multicolumn{3}{|c|}{$\begin{array}{c}\mathrm{Mg} \\
\left(\mathrm{kg} \mathrm{ha}^{-1}\right)\end{array}$} & \multicolumn{3}{|c|}{$\begin{array}{c}S \\
\left(\mathrm{~kg} \mathrm{ha}^{-1}\right)\end{array}$} \\
\hline & Seed & Stover & Total & Seed & Stover & Total & Seed & Stover & Total \\
\hline T1 & 1.05 & 9.98 & 11.03 & 0.33 & 5.47 & 5.80 & 0.33 & 0.45 & 0.78 \\
\hline $\mathbf{T} 2$ & 2.14 & 23.04 & 25.18 & 1.20 & 14.41 & 15.61 & 0.88 & 1.23 & 2.11 \\
\hline T3 & 2.89 & 29.88 & 32.77 & 1.52 & 17.98 & 19.50 & 1.52 & 2.41 & 3.93 \\
\hline $\mathbf{T 4}$ & 3.28 & 31.89 & 35.16 & 1.75 & 19.22 & 20.97 & 1.98 & 3.13 & 5.11 \\
\hline T5 & 2.58 & 26.66 & 29.24 & 1.32 & 15.99 & 17.32 & 1.18 & 2.05 & 3.24 \\
\hline T6 & 2.75 & 27.68 & 30.43 & 1.34 & 16.33 & 17.67 & 1.34 & 2.08 & 3.42 \\
\hline T7 & 2.96 & 29.88 & 32.84 & 1.45 & 17.98 & 19.43 & 1.59 & 2.41 & 4.00 \\
\hline T8 & 3.19 & 30.97 & 34.16 & 1.56 & 18.90 & 20.46 & 1.70 & 2.76 & 4.47 \\
\hline T9 & 2.67 & 28.78 & 31.45 & 1.47 & 16.39 & 17.86 & 1.40 & 2.07 & 3.47 \\
\hline T10 & 2.94 & 29.57 & 32.51 & 1.58 & 17.88 & 19.46 & 1.51 & 2.39 & 3.90 \\
\hline T11 & 2.99 & 30.03 & 33.02 & 1.60 & 18.30 & 19.91 & 1.68 & 2.43 & 4.11 \\
\hline T12 & 3.49 & 32.70 & 36.19 & 1.94 & 19.77 & 21.71 & 2.02 & 3.35 & 5.36 \\
\hline T13 & 3.21 & 31.14 & 34.35 & 1.72 & 18.86 & 20.58 & 1.72 & 2.78 & 4.50 \\
\hline T14 & 3.43 & 32.70 & 36.13 & 1.83 & 19.56 & 21.39 & 1.98 & 3.14 & 5.12 \\
\hline T15 & 3.83 & 35.17 & 39.00 & 2.07 & 21.26 & 23.33 & 2.15 & 3.91 & 6.06 \\
\hline T16 & 4.53 & 40.65 & 45.18 & 2.53 & 24.77 & 27.29 & 2.61 & 4.44 & 7.05 \\
\hline S.Em \pm & 0.16 & 1.45 & 1.46 & 0.05 & 0.90 & 0.87 & 0.08 & 0.11 & 0.16 \\
\hline C.D. $(p=0.05)$ & 0.46 & 4.20 & 4.22 & 0.16 & 2.60 & 2.50 & 0.24 & 0.32 & 0.46 \\
\hline
\end{tabular}

Note: CS: Coconut shell, RDF: Recommended dose of fertilizer, POP: Package of practice

\begin{tabular}{|c|c|c|}
\hline $\mathbf{T}_{1}$ : Absolute Control & $\mathrm{T}_{7}: \mathrm{CS}$ - Biochar @ $6 \mathrm{t} \mathrm{ha}^{-1}$ & T12: CS - Biochar @ $8 \mathrm{t} \mathrm{ha}^{-1}+$ FYM @ $5 \mathrm{t} \mathrm{ha}^{-1}$ \\
\hline$T_{2}: 100: 50: 50 \mathrm{NPK} \mathrm{kg} \mathrm{ha}^{-1}$ (Only RDF) & T8:CS - Biochar@8t ha ${ }^{-1}$ & T \\
\hline $\mathrm{T}_{3}:$ FYM @ $5 \mathrm{tha}^{-1}$ & T9: CS - Biochar@ $@ \mathrm{tha}^{-1}+\mathrm{FYM} @ 5 \mathrm{tha}^{-1}$ & $\mathrm{~T}_{14}: \mathrm{CS}$ - Biochar@ $4 \mathrm{tha} \mathrm{h}^{-1}+\mathrm{FYM} @ 10 \mathrm{tha}^{-1}$ \\
\hline $\mathrm{T}_{4}:$ FYM @10 t ha ${ }^{-1}($ POP $)$ & T10: CS - Biochar@ @ t ha ${ }^{-1}+\mathrm{FYM} @ 5 \mathrm{tha}^{-1}$ & $\mathrm{~T}_{15}: \mathrm{CS}$ - Biochar@6t ha-1 +FYM @10 t ha ${ }^{-1}$ \\
\hline $\mathrm{T}_{5}:$ CS - Biochar @ $2 \mathrm{tha}^{-1}$ & $\mathrm{~T}_{11}$ : CS - Biochar@6 tha ${ }^{-1}+\mathrm{FYM} @ 5 \mathrm{tha}^{-1}$ & $\mathrm{~T}_{16}: \mathrm{CS}$ - Biochar @ $8 \mathrm{t} \mathrm{ha}^{-1}+\mathrm{FYM} @ 10 \mathrm{t} \mathrm{ha}^{-1}$ \\
\hline $\mathrm{T}_{6}:$ CS - Biochar@ 4 t ha ${ }^{-1}$ & & \\
\hline
\end{tabular}


Table.5 Residual effect of levels of biochar and FYM on uptake of micronutrients by seed and stover of green gram at harvest

\begin{tabular}{|c|c|c|c|c|c|c|c|c|c|c|c|c|}
\hline \multirow[t]{2}{*}{ Treatments } & \multicolumn{3}{|c|}{$\begin{array}{c}\text { Fe } \\
\left(\mathrm{g} \mathrm{ha}^{-1}\right)\end{array}$} & \multicolumn{3}{|c|}{$\begin{array}{c}\text { Mn } \\
\left(\mathrm{g} \mathrm{ha}^{-1}\right)\end{array}$} & \multicolumn{3}{|c|}{$\begin{array}{c}\mathbf{Z n} \\
\left(\mathrm{g} \mathrm{ha}^{-1}\right)\end{array}$} & \multicolumn{3}{|c|}{$\begin{array}{c}\mathbf{C u} \\
\left(\mathrm{g} \mathrm{ha}^{-1}\right)\end{array}$} \\
\hline & Seed & Stover & Total & Seed & Stover & Total & Seed & Stover & Total & Seed & Stover & Total \\
\hline T1 & 27.01 & 205.95 & 232.96 & 2.40 & 30.40 & 32.81 & 4.90 & 13.61 & 18.51 & 2.40 & 3.37 & 5.77 \\
\hline $\mathbf{T} 2$ & 53.08 & 392.60 & 445.68 & 4.93 & 63.35 & 68.28 & 10.21 & 32.25 & 42.47 & 5.09 & 9.22 & 14.31 \\
\hline T3 & 61.22 & 465.24 & 526.46 & 6.00 & 84.80 & 90.81 & 13.07 & 41.08 & 54.15 & 7.30 & 12.87 & 20.17 \\
\hline $\mathbf{T 4}$ & 69.34 & 498.02 & 567.36 & 6.63 & 98.73 & 105.36 & 15.67 & 45.61 & 61.28 & 8.54 & 15.05 & 23.59 \\
\hline T5 & 68.31 & 448.38 & 516.68 & 6.29 & 80.13 & 86.42 & 11.90 & 31.77 & 43.67 & 7.23 & 12.48 & 19.71 \\
\hline T6 & 69.99 & 463.34 & 533.33 & 6.57 & 83.16 & 89.73 & 12.75 & 32.37 & 45.12 & 7.91 & 12.84 & 20.76 \\
\hline $\mathbf{T 7}$ & 72.00 & 476.95 & 548.95 & 6.74 & 90.86 & 97.60 & 13.46 & 36.55 & 50.00 & 8.68 & 14.17 & 22.86 \\
\hline T8 & 75.04 & 501.60 & 576.64 & 7.20 & 94.48 & 101.68 & 15.51 & 39.43 & 54.94 & 10.27 & 15.35 & 25.62 \\
\hline T9 & 70.96 & 461.24 & 532.20 & 6.74 & 82.73 & 89.47 & 13.56 & 37.76 & 51.32 & 7.88 & 13.74 & 21.63 \\
\hline T10 & 74.64 & 474.30 & 548.94 & 7.06 & 86.78 & 93.83 & 14.30 & 40.87 & 55.17 & 8.52 & 14.33 & 22.86 \\
\hline T11 & 75.28 & 482.15 & 557.43 & 7.25 & 93.71 & 100.95 & 14.94 & 44.33 & 59.27 & 8.81 & 14.59 & 23.40 \\
\hline T12 & 80.91 & 529.46 & 610.37 & 7.93 & 103.28 & 111.21 & 16.47 & 48.25 & 64.72 & 9.52 & 17.31 & 26.83 \\
\hline T13 & 78.31 & 503.50 & 581.81 & 7.62 & 95.79 & 103.41 & 16.42 & 47.35 & 63.77 & 10.37 & 16.13 & 26.49 \\
\hline T14 & 79.99 & 520.80 & 600.80 & 8.06 & 100.37 & 108.44 & 17.31 & 50.91 & 68.22 & 10.10 & 16.74 & 26.84 \\
\hline T15 & 84.76 & 549.49 & 634.25 & 8.47 & 113.93 & 122.40 & 18.60 & 54.06 & 72.67 & 11.17 & 18.18 & 29.35 \\
\hline T16 & 94.80 & 609.93 & 704.73 & 9.93 & 129.72 & 139.65 & 21.65 & 62.03 & 83.69 & 12.79 & 21.20 & 33.99 \\
\hline S.Em \pm & 2.67 & 20.84 & 21.35 & 0.34 & 4.12 & 4.10 & 0.51 & 2.34 & 2.46 & 0.44 & 0.73 & 1.09 \\
\hline C.D. $(p=0.05)$ & 7.71 & 60.20 & 61.67 & 0.99 & 11.89 & 11.84 & 1.48 & 6.77 & 7.12 & 1.27 & 1.03 & 3.14 \\
\hline
\end{tabular}

Note: CS: Coconut shell, RDF: Recommended dose of fertilizer, POP: Package of practice

\begin{tabular}{|c|c|c|}
\hline $\mathbf{T}_{1}$ : Absolute Control & $\mathrm{T}_{7}: \mathrm{CS}$ - Biochar @ $6 \mathrm{t} \mathrm{ha}^{-1}$ & 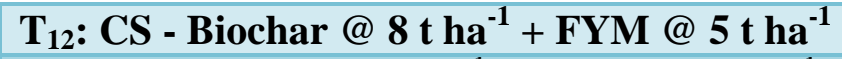 \\
\hline$T_{2}: 100: 50: 50 \mathrm{NPK}_{\mathrm{kg} \mathrm{ha}}^{-1}$ (Only RDF) & T: CS - Biochar@8 tha ${ }^{-1}$ & T \\
\hline $\mathbf{T}_{3}:$ FYM @ 5 t ha $^{-1}$ & T9: CS - Biochar@ $2 \mathrm{tha}^{-1}+\mathrm{FYM} @ 5 \mathrm{tha}^{-1}$ & T14: CS - Biochar@ 4 t ha ${ }^{-1}+$ FYM @ 10 tha $^{-1}$ \\
\hline $\mathrm{T}_{4}:$ FYM @ $10 \mathrm{t} \mathrm{ha}^{-1}(\mathrm{POP})$ & $\mathrm{T}_{10}: \mathrm{CS}$ - Biochar@ $4 \mathrm{tha}^{-1}+\mathrm{FYM} @ 5 \mathrm{tha}^{-1}$ & 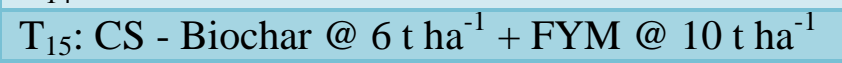 \\
\hline $\mathbf{T}_{5}:$ CS - Biochar @ $2 \mathrm{tha}^{-1}$ & $\mathrm{~T}_{11}$ : CS - Biochar@6 tha- $\mathrm{h}^{-1}+\mathrm{FYM} @ 5 \mathrm{tha}^{-1}$ & T16: CS - Biochar@8t ha- ${ }^{-1}+\mathrm{FYM} @ 10 \mathrm{tha}^{-1}$ \\
\hline $\mathrm{T}_{6}:$ CS - Biochar@ $4 \mathrm{tha}^{-1}$ & & \\
\hline
\end{tabular}


Fig.1 Per cent increase in seed and stover yield due residual effect of biochar and FYM as compared to POP treatment $\left(\mathrm{T}_{4}\right)$ under green gram cultivation

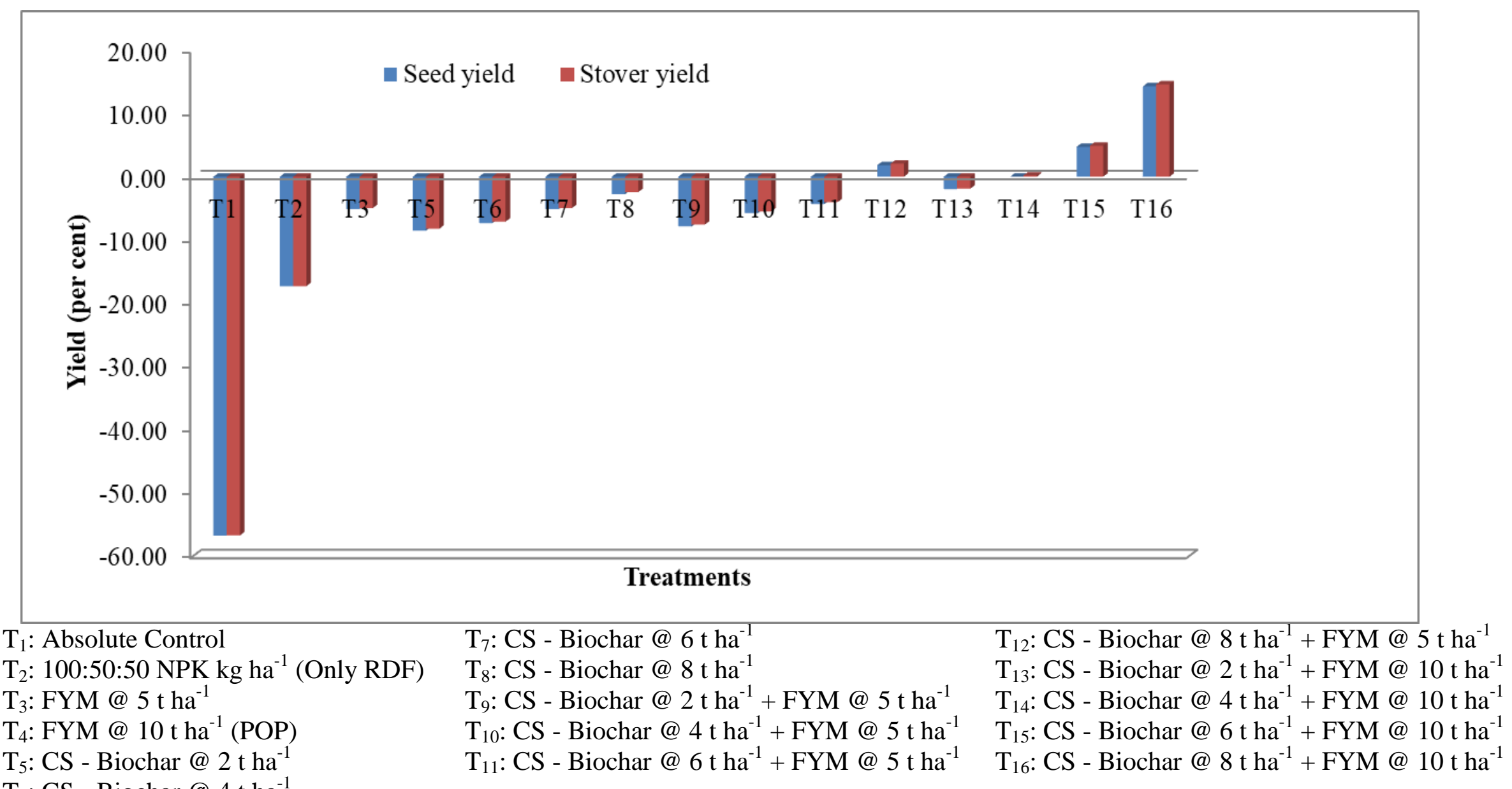


Residual effect of CS-biochar @ $8 \mathrm{t} \mathrm{ha}^{-1}+$ FYM @ $10 \mathrm{t} \mathrm{ha}^{-1}+$ RDF treatment showed significantly higher seed and stover yields 871 and $1708 \mathrm{~kg} \mathrm{ha}^{-1}$, respectively and followed by CS-biochar @6 t ha ${ }^{-1}$ +FYM @ $10 \mathrm{t} \mathrm{ha}^{-1}+\operatorname{RDF}$ (798.0 and $1563 \mathrm{~kg} \mathrm{ha}^{-1}$, respectively) as compared to $\mathrm{T}_{4}(\mathrm{RDF}+\mathrm{FYM}$ (a) $\left.10 \mathrm{t} \mathrm{ha}^{-1}\right)\left(762.0\right.$ and $1490 \mathrm{~kg} \mathrm{ha}^{-1}$, respectively) and $\mathrm{T}_{2}$ (RDF alone) treatment (630.0 and $1232.0 \mathrm{~kg} \mathrm{ha}^{-1}$, respectively), lower seed and stover yields was obtained in absolute control treatment (329 and $644 \mathrm{~kg}$ ha $^{-1}$, respectively).

The yield data indicated a significant influence of residual biochar and FYM on green gram. This could be mainly attributed by nutrients present in the soil and in the biochar and FYM, which were made available to the crop. Balanced supply of nutrients might have enabled green gram crop to produce higher pod bearing auxiliary branches and higher number of pods per plant. Yooyen et al., (2015) reported that addition of biochar to soybean increased the number of seeds per plant. Mirl et al., (2013) reported that an increase in seed yield and haulm yield was observed in the biochar treatment as compared to no biochar treatment. The better performance of green gram crop might be due to the presence of bacterial activity which helps in increasing the phosphorus availability in soil, thereby improving plant growth and yield (Viruell et al., 2014). The RDF applied along with biochar increased the availability of nutrients to plants by performing different mechanisms, which in turn increased the yields. Along with increasing biological nitrogen fixation, biochar also significantly improved biomass production and yield of common beans. Such responses confirmed earlier results with moong bean (Rondon et al., 2007). Bandara et al., (2015) observed increased the crop growth and yield by combined application of biochar and FYM. The timely availability of nitrogen with the interaction effect of biochar and FYM mineralization might be responsible for the production of highest seed and stover yield. The superiority of coconut shell biochar applied treatments over RDF alone was due to biochars was being protected from further decomposition thereby it could supply longer and steady source of macro and micronutrients. However, in present study residual biochar and FYM applied in combination recorded higher yield of seed and stover in the treatment $\mathrm{T}_{16}$ (CS-biochar @ $8 \mathrm{t} \mathrm{ha}^{-1}+$ FYM @ $\left.10 \mathrm{t} \mathrm{ha}^{-1}+\mathrm{RDF}\right)$. Which showed the percentage efficiency of 14.30 and 14.63 per cent increase in grain and stover yield of green gram respectively over treatment $\mathrm{T}_{4}\left(\mathrm{RDF}+\mathrm{FYM} 10 \mathrm{t} \mathrm{ha}^{-1}\right)$ (Fig. I).

\section{Nutrient uptake}

Significantly higher primary, secondary and micronutrients uptake by green gram was noticed in $\mathrm{T}_{16}$ which received CS-biochar@8 $\mathrm{t} \mathrm{ha}^{-1}+$ FYM@10 t ha ${ }^{-1}+$ RDF (Table 3 to $5)$. The reason for higher uptake of primary nutrients under high doses of biochar and FYM might be due to the positive effects of biochar on crop growth, along with positive effects on nutrient $(\mathrm{P}, \mathrm{K}, \mathrm{Ca}$ and $\mathrm{Mg}$ ) uptake by crop plants and the availability of soil $\mathrm{N}$, $\mathrm{P}, \mathrm{K}, \mathrm{Ca}$ and $\mathrm{Mg}$. Increase in $\mathrm{pH}$ of acidic soil may decrease Al activity, hence better root growth and nutrient uptake can be expected. Nutrient uptake is a function of nutrient content and biomass production. Increased rate of application of biochar increased biomass production which obviously increased nutrient uptake. Chan et al., (2007) observed an increase in the uptake of $\mathrm{N}$ at higher levels of biochar. Yao et al., (2013) reported that the bioavailability and plant uptake of primary nutrients increased in response to biochar application, particularly in the presence of added fertilizer. Luca et al., (2009) stated that biochar added to soil with an organic $\mathrm{N}$ source yielded an increase in net 
nitrification and improves the nitrogen availability to the plants. Similar findings were reported by Eazhilkrishna et al., (2017).

It is concluded from the field investigation the residual impact of biochar and FYM with recommended dose fertilizers for green gram, resulted the most favourable physical, physico-chemical, chemical and biological properties of soil. The coconut shell biochar is a high $\mathrm{C}: \mathrm{N}$ ratio organic material, that could be reduced by the application of FYM. Since, FYM is a good source of microorganisms and hastens the better mineralization rate and increased the efficiency of biochar in soil. Further, higher uptake of nutrients, growth and yield of succeeding green gram crop was obtained due to residual effect of biochar and FYM. Overall, the present study indicated that, residual effect of applied biochar and FYM with application of recommended inorganic fertilizers for higher growth and yield, reflecting and confirming the improved nutrient availability to pulse crop as compared to alone application of biochar, FYM and fertilizers.

\section{References}

Bandara, T., Herath, I., Prasanna, K., Mihiri, S., Gamini, S., Nishanta, R., Meththika, V. and YONG, S. O. 2015. Role of woody biochar and fungal bacterial coinoculation on enzyme activity and metal immobilization in serpentine soil. J. Soils Sediments. 10(7): 43-68.

Chan, K. Y., Zwiteten, L. V., Meszaros, I., Downie, A. and Joseph, S. 2007. Agronomic values of green waste biochar as a soil amendment. Soil Res. 45: 629-634.

Eazhilkrishna, N., Thilagavathi, T. and Baskar, M. 2017. Eco friendly utilization of nutrient enriched biochar from sugar industry wastes and its effect on yield and NPK uptake by hybrid maize grown in vertisol. Int. J. Agric. Sci. 9(31): 4456-4459.

Glaser, B., Lehmann, J. and Zech, W. 2002. Ameliorating physical and chemical properties of highly weathered soils in the tropics with charcoal - A review. Biol. Fertil. Soils. 35: 219-230.

Gomez, K. A. and Gomez, A. A. 1984 Statistical Procedures for Agric. Res., 2nd Ed. John Wiley and Sons, New York.

Lehmann, J., Gaunt, J. and Rondon, M. 2006. Bio-char sequestration in terrestrial ecosystems: A review. Mitig. Adapt. Strat. Gl. 11: 403-427.

Luca, T. H. D., Mackenzie, M. D. and Gundale, M. J. 2009. Biochar effects on soil nutrient transformations. In Biochar for environmental management Science and Technology., pp. 251-270.

Mirl, A. H., Lal, S. B., Salmani, M., Abid, M. and Khan, I. 2013. Growth, yield and nutrient content of blackgram (Vigna mungo) as influenced by levels of phosphorus, sulphur and phosphorus solubilizing bacteria. SAARC J. Agri. 11(1): 1 - 6 .

Muthaura, C., David, M. Musyimi, Joseph, A., Ogur and Samuel, V. Okello. 2010. Effective microorganisms and their influence on growth and yield of pigweed (Amaranthus dubians). $J$. Agric. Biol. Sci. 5(1): 17-22.

Rondon, M. A., Lehmann, J., Ramirez, J. and Hurtado, M. 2007. Biological nitrogen fixation by common beans increases with biochar additions. Biol. Fertil. Soils. 43(6): 699-708.

Viruell, E., Erazzu, L. E., Martinez, Calsina, L., Ferrero, M. A., Lucca, M. E. and Sineriz, F. 2014. Inoculation of maize with phosphate solubilizing bacteria effect on plant growth and yield. J. Soil Sci. Plant Nutr. 14(4): 819-831.

Warnock, D. D., Lehmann, J., Kuyper, T. W. and Rillig, M. C. 2007. Mycorrhizal 
responses to biochar in soil - concepts and mechanisms. Plant Soil. 300: 9-20.

Widowati, Astutik, Sumiati, A. and Fikrinda, W. 2017. Residual effect of potassium fertilizer and biochar on growth and yield of maize in the second season. $J$. Degrade. Min. Land Manage. 4(4): 881889.

Wisnubroto, E. I., Utomo, W. H. and Indrayatie, E. R. 2016. Residual effect of biochar on growth and yield of red chilli (Capsicum annum L.). Soil Sci.
37: 29-38.

Yao, M., Angui, P. K. T., Konate, S. and Tondoh, J. E. 2013. Effects of land use types on soil organic carbon and nitrogen dynamics in Mid-West Cote d'Ivoire. European J. Sci. Res. 40(2): 211-222.

Yooyen, J., Wijitkosum, S. and Sriburi, T. 2015. Increasing yield of soybean by adding biochar. J. Environ. Res. Dev. 9(4): 1066-1074.

\section{How to cite this article:}

Arunkumar, B. R. and Thippeshappa, G. N. 2020. Residual Effect of Levels of Biochar and FYM on Growth, Yield and Nutrient Uptake by Green Gram (Vigna radiate L.) Crop in Sandy Loam Soil. Int.J.Curr.Microbiol.App.Sci. 9(02): 695-707.

doi: https://doi.org/10.20546/ijcmas.2020.902.085 